\title{
Adverse Event End Relative to Reference Period
}

National Cancer Institute

\section{Source}

National Cancer Institute. Adverse Event End Relative to Reference Period. NCI

Thesaurus. Code C83203.

A characterization of the relationship of an adverse event conclusion point in time to a reference point in time. 